\section{ORIGINAL RESEARCH}

Y. Jinhu

D. Jianping

L. Xin

Z. Yuanli

\title{
Dynamic Enhancement Features of Cavernous Sinus Cavernous Hemangiomas on Conventional Contrast-Enhanced MR Imaging
}

\begin{abstract}
BACKGROUND AND PURPOSE: The reported MR imaging characteristics of cavernous sinus cavernous hemangiomas ( $\mathrm{CSCHs}$ ) in the literature are nonspecific. The purpose of our study was to explore dynamic enhancement features of $\mathrm{CSCH}$ s on conventional contrast-enhanced MR imaging and to correlate these features with histopathologic subtypes.
\end{abstract}

\begin{abstract}
MATERIALS AND METHODS: Twenty-one patients (8 male and 13 female; age range, 13-63 years; average age, 42.6 years) with surgically confirmed $\mathrm{CSCHs}$ were retrospectively investigated. Preoperative MR study was performed in all cases, consisting of T1-weighted axial imaging, T2-weighted axial imaging, T1-weighted sagittal imaging, and contrast-enhanced T1-weighted axial, sagittal, and coronal images.
\end{abstract}

\begin{abstract}
RESULTS: There were $4.8 \%(1 / 21)$ that showed homogeneous enhancement on all 3 contrast-enhanced sequences, whereas $95.2 \%$ (20/21) demonstrated heterogeneous enhancement on the first contrast-enhanced sequence. Among the 20 lesions, on subsequent contrast-enhanced sequences, $55.0 \%(11 / 20)$ showed homogeneous enhancement, whereas $35.0 \%(7 / 20)$ of lesions showed progressive contrast "filling in." The remaining 10\% (2/20) exhibited no apparent enhancement changes. The 95.2\% (20/21) of lesions with heterogeneous enhancement on the first contrast-enhanced sequence correlated with type B or type C pathologic findings, whereas $4.8 \%(1 / 21)$ with homogeneous enhancement correlated with type A pathologic findings. Among the 20 type $\mathrm{B}$ or type $\mathrm{C}$ lesions, $80 \%(16 / 20)$ achieved total or near-total resection.
\end{abstract}

CONCLUSION: Progressive contrast "filling in" in the tumors on conventional contrast-enhanced MR images can aid in differentiating between cavernous sinus lesions and suggest the diagnosis of cavernous hemangiomas.

C avernous sinus cavernous hemangiomas (CSCHs) are rare vascular malformations, accounting for less than $1 \%$ of all parasellar masses. ${ }^{1,2}$ Because of their tendency to bleed profusely during surgery, CSCHs require a different surgical approach and technique. ${ }^{3-5}$ However, the rate of misdiagnosis is as high as $38.9 \%{ }^{6}$ Although the primary diagnostic tool for CSCHs is MR imaging, there are only scattered reports on their MR characteristics in the literature. The value of these reported MR characteristics is limited in diagnosis and differential diagnosis. However, as a specific characteristic of cavernous hemangiomas in extracranial organs, contrast "filling in" with CSCHs on dynamic MR imaging or CT has not been studied up to now. Our paper is a retrospective review of 21 patients with CSCHs surgically treated at our institution from 2001 through 2007, with particular focus on exploring new MR diagnostic characteristics for this lesion.

\section{Materials and Methods}

For our study, which was approved by our institutional ethics committee, we retrospectively enrolled 21 patients with surgically confirmed $\mathrm{CSCH}$ ( 13 female and 8 male patients, with a female/male ratio of 1.6:1; age range, 13-63 years, with an average age of 42.6 years), including 2 patients who were operated on in outside hospitals and

Received June 7, 2007; accepted after revision September 18

From the Department of Neuroradiology and Department of Neurosurgery, Beijing Tiantan Hospital, Capital Medical University, Beijing, China.

Please address correspondence to Dai Jianping, Department of Neuroradiology, Beijing Tiantan Hospital, Capital Medical University, Tiantan Xili No.6, Beijing, 100050, China; e-mail: yjh2005bj@sina.com

DOI 10.3174/ajnr.A0845 referred to our institution for a second operation. The time of onset of symptoms to the admission ranged from 1 month to 20 years, with an average of 2.0 years. The presenting signs and symptoms were headache in $52.4 \%$, diplopia in $19.0 \%$, visual impairment in $23.8 \%$, facial numbness in $23.8 \%$, menolipsis in $14.2 \%$, primary optic nerve atrophy in $4.7 \%$, cranial nerve III palsy in $9.5 \%$, cranial nerve $\mathrm{V}$ palsy in $23.85 \%$, and cranial nerve VI palsy in $9.5 \%$.

All patients underwent MR imaging examination preoperatively. The MR imaging protocol consisted of T1- and T2-weighted axial imaging; T1-weighted sagittal imaging; and contrast-enhanced T1-weighted axial, sagittal, and coronal imaging. Ten patients underwent MR imaging on a 3T superconducting MR scanner (Signa; GE Healthcare, Milwaukee, Wis). T1-weighted flair sequences (TR, 1971.86-2143.22; TE, 19.5019.70) and fast spin-echo (FSE) T2-weighted sequences (TR, 4900; TE, 116.61) were performed. Six patients underwent MR imaging on another 3T superconducting MR scanner (Magnetom, Trio; Siemens, Erlangen, Germany). T1-weighted turbo inversion recovery sequences (TR, 1680.00-2520.00; /TE, 12.00-19.00) and T2-weighted turbo spin-echo scan (TR, 5800.00; TE, 103.00) were performed. The remaining 5 patients underwent MR imaging on a 1.5T superconducting MR scanner (Visart; Toshiba, Japan). Spin-echo T1-weighted sequences (TR, 300.00-560.00; TE, 12.00-15.00) and FSE T2-weighted sequence (TR, 4800.00; TE, $100.00)$ were performed. The parameters used in all the patients were as follows: section thickness, 5- to 6-mm; section gap, 5.0 to 7.8-mm; FOV, 220 or 240 -mm; matrix, 256 to $512 \times 208$ to 512 . In addition, thin section thickness and reduced section gap were adopted in the 2 lesions in which the sizes were less than $2.5 \mathrm{~cm}$. Gadopentetate dimeglumine (Magnevist; Bayer HealthCare Pharmaceuticals, Wayne, NJ) was injected manually as fast as possible through an antecubital vein with a 22-gauge needle at a dose of $0.2 \mathrm{~mL} / \mathrm{kg}$ of body weight by 2 registered nurses. The injection 

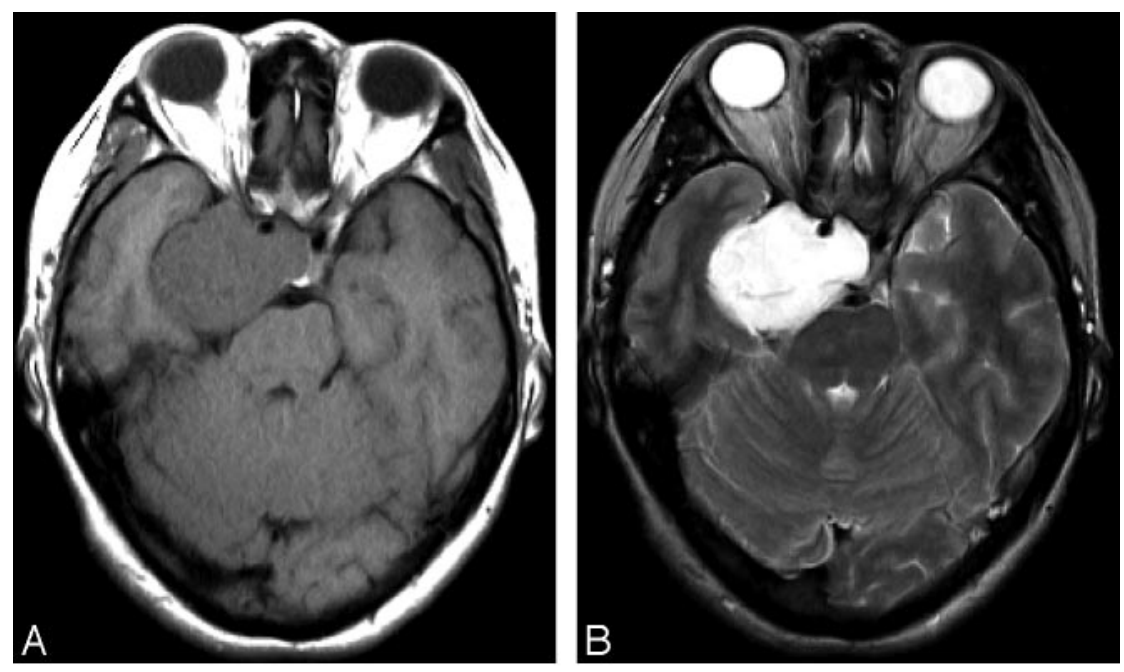

Fig 1. A 52-year-old woman presented with a 1-year history of blurred vision in both eyes. A, T1-weighted axial image exhibits a hypointense dumbbell-shaped mass involving both sellar and parasellar regions. $B$, The mass demonstrates marked hyperintensity on T2-weighted axial imaging, and encasement of the right internal carotid artery can be seen. $C$, Strong homogeneous enhancement can be found in the mass on postcontrast T1-weighted axial imaging. D, Microphotograph of surgical specimen reveals large numbers of thin-walled vascular sinusoids, with a single layer of endothelium-lined capillaries, and scanty connective tissue (type A pathologic features; hematoxylin and eosin [H\&E], original magnification, $\times 40$ ).

sification, further generalized their pathologic patterns into type $\mathrm{A}, \mathrm{B}$, or $\mathrm{C}$.

If different opinions occurred between the 2 neuroradiologists or between the 2 neuropathologists, a conclusion was reached by consensus. Finally, the results of surgical treat-
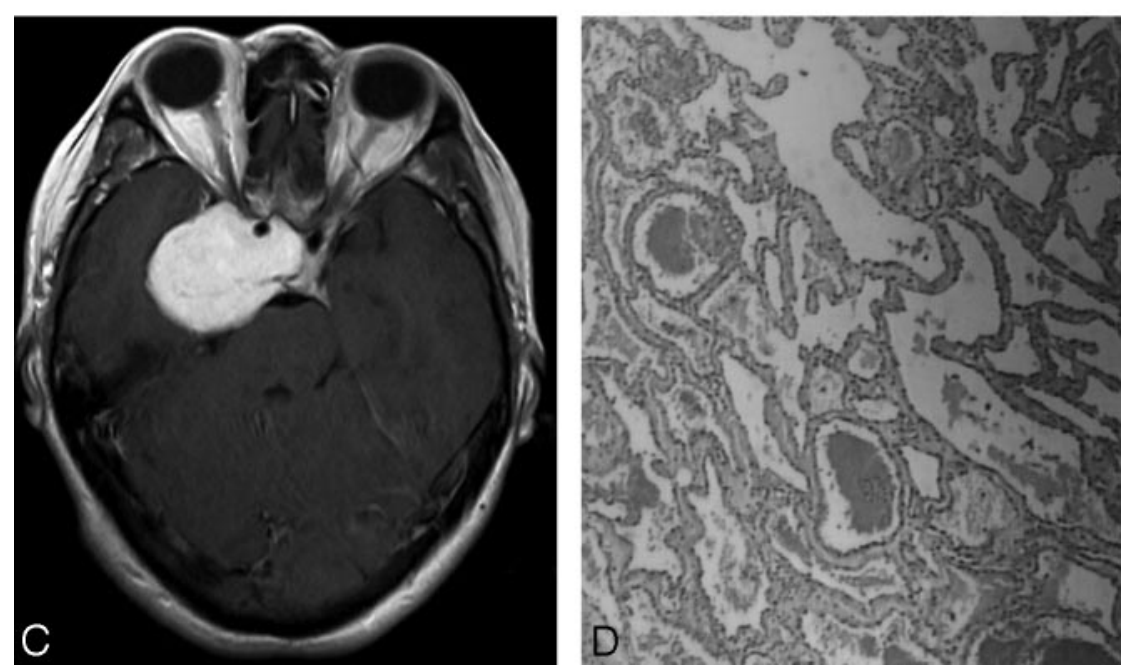
ment were analyzed and correlated with pathologic subtypes and MR findings.

\section{Results}

Twenty-one lesions measured from 1.6 to $7.4 \mathrm{~cm}$ (average, $4.3 \mathrm{~cm}$ ) with $71.4 \%$ $(15 / 21)$ of lesions located to the right side and $28.6 \%(6 / 21)$ to the left side. On T1weighted images, $57.1 \%(12 / 21)$ of lesions were isointense to white matter, whereas the other $42.9 \%$ (9/21) were hypointense (Fig 1A). On T2-weighted images, 90.5\% (19/21) of lesions showed marked homogeneous hyperintensity (Fig $1 B$ ) with 9.5\% (2/21) demonstrating mixed intensity. A total of $85.7 \%(18 / 21)$ of lesions were dumbbell-shaped, pro-

rate was nearly constant, and it can be calculated by dividing the volume of contrast material administered (in milliliters) by the length of time which the contrast material was injected (in seconds). In our series, the injection rate was approximately $1.6 \mathrm{~mL} / \mathrm{s}$. The interval between the initiation of intravenous injection and the start of the postcontrast T1weighted image acquisition ranged from 35 to 60 seconds. The range of scanning time was 1:01 to 1:57 minutes for the first postcontrast sequence, 1:01 to 1:57 minutes for the second, and 1:01 to 1:56 minutes for the third, respectively. The 3 sequences resulted in 2 interscan delays, and their ranges were 0:05 to 0:40 minutes and 0:05 to 0:50 minutes, respectively.

Two neuroradiologists, who were not informed of the clinical and pathologic findings, independently reviewed the size (maximum diameter), signal intensity characteristics, morphology, ipsilateral internal carotid artery, enhancing pattern, and progressive contrast "filling in" in these lesions on MR images.

On pathologic examination, $\mathrm{CSCH}$ s are classified into 3 subtypes by Zhou et $\mathrm{al}^{6}$ and Yao et al. ${ }^{7}$ Type A is composed of large numbers of thin-walled vascular sinusoids, and between the large lumens of the vessels, connective tissue is scanty. Type B contains ample solid parenchyma and well-formed vasculature and connective tissue. Type $\mathrm{C}$ is composed of both type A and type B compositions. ${ }^{6,7}$ Another 2 neuropathologists, who were blinded to the MR findings, evaluated the 21 surgical specimens and their histopathologic characteristics, confirmed the final pathologic diagnoses, and according to the clas- truding into the sellar turcica, whereas $14.3 \%$ (3/21) were round-shaped. Internal carotid arteries were encircled without significant stenosis by the lesions in $85.7 \%(18 / 21)$ of cases. A total of $4.8 \%(1 / 21)$ showed homogeneous enhancement on all 3 contrast-enhanced sequences (Fig 1C), whereas 95.2\% (20/21) demonstrated heterogeneous enhancement on the first contrast-enhanced sequence (Fig. $2 A$, Fig $3 A$ ). Among the 20 lesions, on subsequent contrast-enhanced sequences, $55.0 \%(11 / 20)$ showed homogeneous enhancement (Fig 2B), whereas $35.0 \%(7 / 20)$ of lesions showed progressive contrast "filling in" (Fig 3B,C). In addition, 10\% (2/20) exhibited no apparent enhancement changes.

The 95.2\% (20/21) of lesions with heterogeneous enhancement on the first contrast-enhanced sequence correlated with type B (Fig 3D) or C (Fig. 2C) pathologic findings, whereas $4.8 \%(1 / 21)$ with homogeneous enhancement correlated with type A pathologic findings (Fig. 1D). Among the 20 type $\mathrm{B}$ and C lesions, $80 \%(16 / 20)$ achieved total or near total resection.

\section{Discussion}

CSCHs are, in fact, hamartomas or malformations of the microcirculation, rather than vascular neoplasms. ${ }^{2}$ When growing to the point that they start to compress neighboring structures, these lesions behave like a real tumor. As reported previously, CSCHs also had a propensity to occur in middleaged women in our series. ${ }^{6}$ Among these 21 patients, the main 

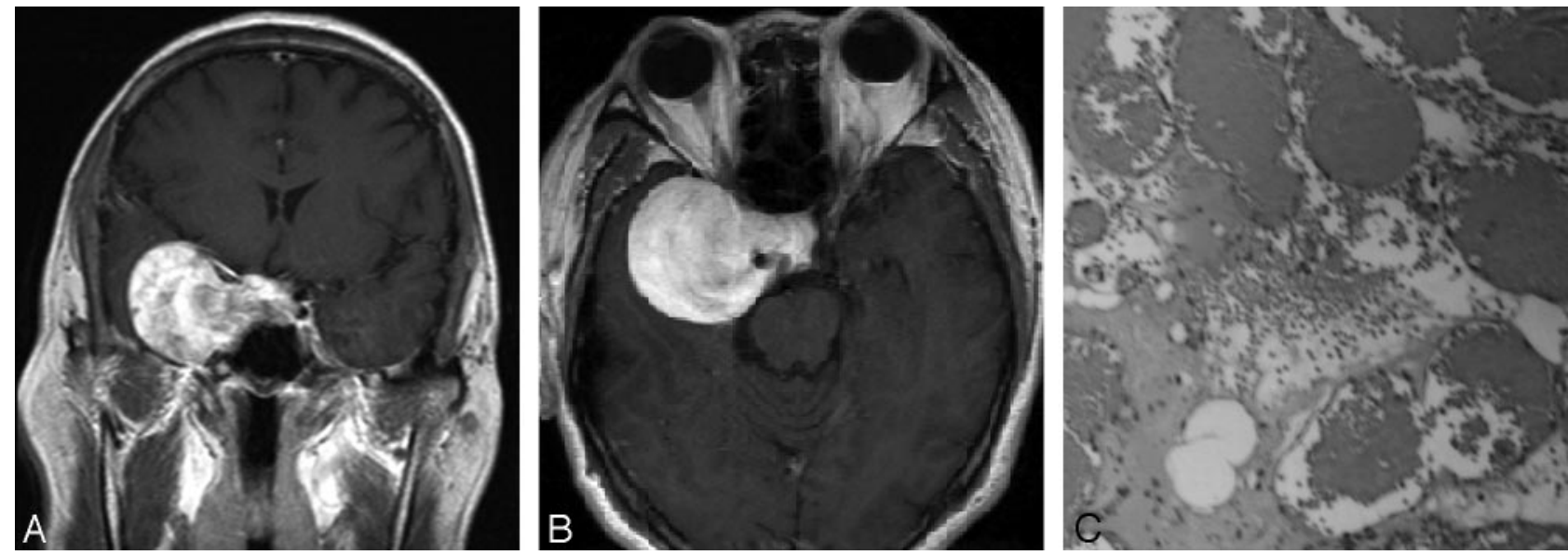

Fig 2. A 48-year-old man was admitted with an 8-year history of intermittent headache. A, Postcontrast T1-weighted coronal image shows a heterogeneously enhanced mass in the right parasellar region. $B$, On subsequent T1-weighted axial image, the mass exhibits homogeneous enhancement. $C$, Microphotograph of surgical specimen shows a combination of both types $A$ and $B$ composition (type $C$ pathologic features; H\&E, original magnification, $\times 40$ ).
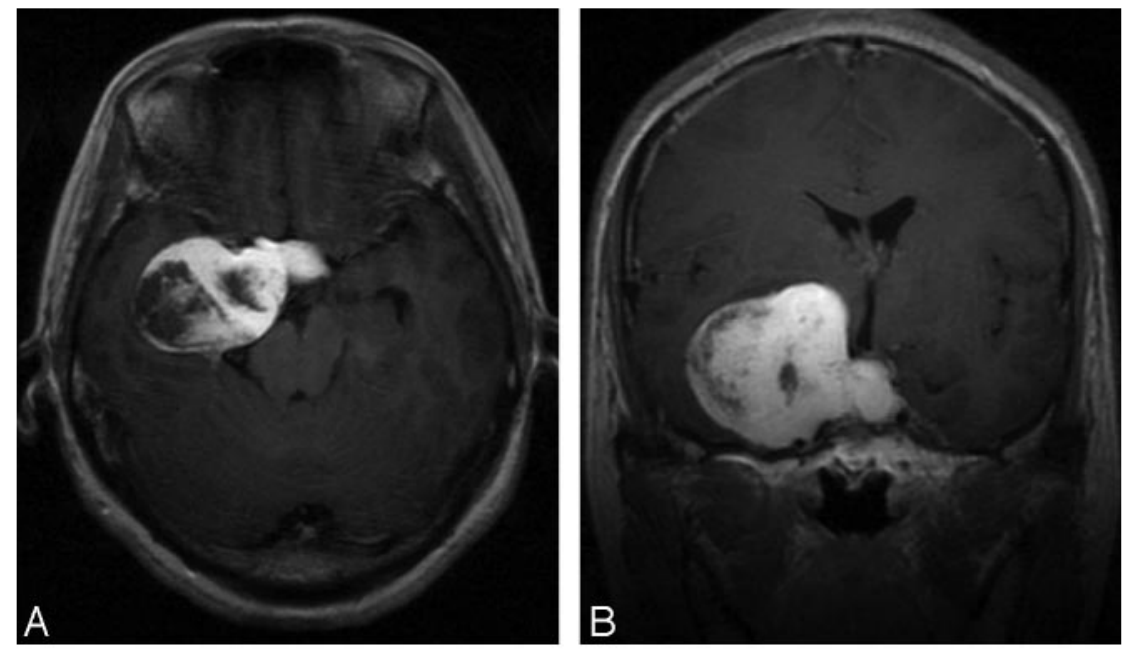

Fig 3. A 45-year-old woman presented with right facial numbness of 1-year duration. A, Contrast-enhanced T1weighted axial image shows inhomogeneous enhancement of a mass in the right parasellar region. $B$, On subsequent T1-weighted coronal image, progressive contrast "filling in" in the mass can be found. C, Postcontrast delayed T1weighted axial image obtained 6 minutes after intravenous administration of contrast material reveals nearly homogenous enhancement in the mass. $D$, Microphotograph of surgical specimen shows ample solid parenchyma and wellformed vasculature and connective tissue (type B pathologic features; H\&E, original magnification, $\times 40$ ).
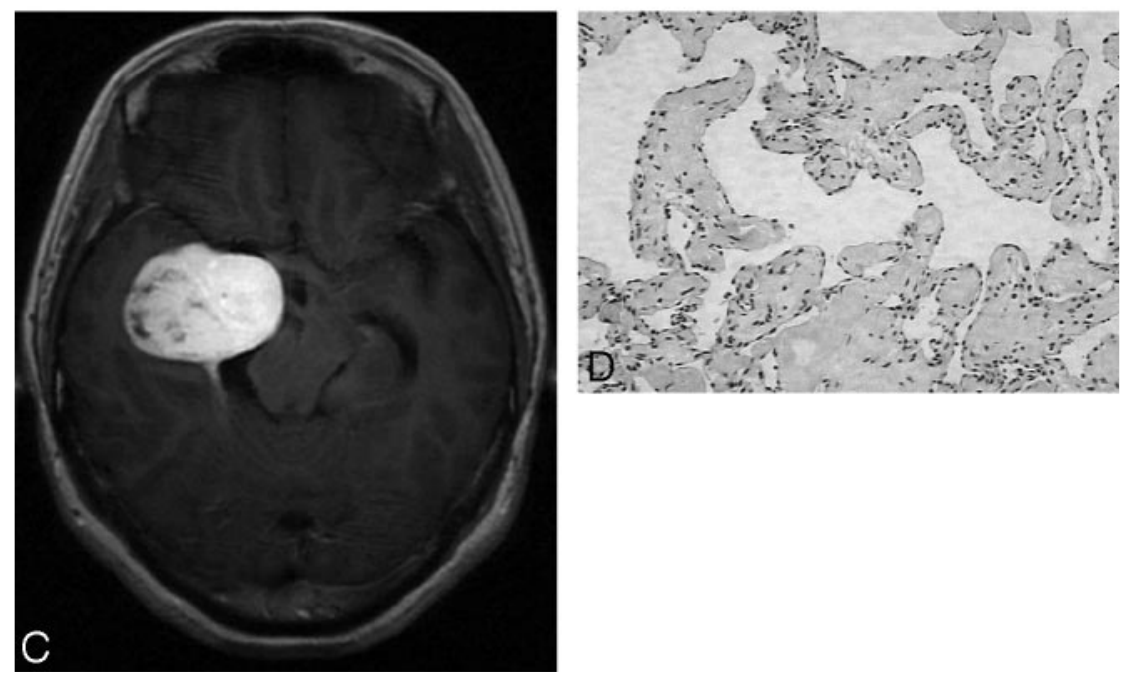

clinical manifestations included visual loss, diplopia, headache, facial numbness, and extraocular muscle palsy. In general, the CSCHs occurred insidiously and deteriorated gradually. In pregnant women, these symptoms may exacerbate. ${ }^{8}$ However, it is difficult to differentiate CSCHs from the common cavernous sinus tumors, such as meningioma, neurinoma, and pituitary adenoma from a clinical standpoint.
The usefulness of MR imaging of CSCHs is well known. In our series, the similarity of our cases in MR imaging appearance to those reported in literature is remarkable. CSCHs are isointense or hypointense relative to gray matter on T1weighted images and markedly hyperintense (nearly as bright as CSF) on T2-weighted images. Encasement of ipsilateral internal carotid artery without stenosis of its lumen can be found 
in most cases. Most of the larger lesions are dumbbell-shaped. In the current literature, high signal intensity on T2-weighted images with strong homogeneous enhancement describes the MR characteristics of CSCHs. ${ }^{9}$ When these characteristics present, a diagnosis of $\mathrm{CSCH}$ is strongly suggested. ${ }^{10}$ However, they are actually nonspecific because angioblastic meningiomas and neurilemomas may have similar MR appearances, and it is quite difficult to differentiate them relying solely on the above-mentioned MR features. ${ }^{11}$

Fortunately, as a pathognomonic MR feature of cavernous hemangiomas in the liver revealed by the traditional dynamic contrast-enhanced T1-weighted spin-echo study, progressive contrast "filling in" was frequently found on conventional contrast-enhanced T1-weighted sequences in our series. ${ }^{12,13}$ Actually, they revealed the dynamic enhancement of the lesions to a great extent because axial, sagittal, and coronal images were obtained in succession after intravenous injection of contrast. One case showed homogeneous enhancement on all 3 contrast-enhanced T1-weighted sequences, and on pathologic examination, it belonged to type A. In our opinion, this can be explained by their pathologic findings. Because type A is composed of large numbers of thin-walled vascular sinusoids, it requires a relatively shorter time for type A to achieve homogeneous enhancement on MR. Also, when our enhancement sequences were performed, a homogeneous contrast enhancement in the tumor was obtained in all 3 sequences. The 11 lesions that showed inhomogeneous enhancement on the first contrast-enhanced sequence and obtained homogeneous enhancement on subsequent contrast-enhanced sequences were type $\mathrm{B}$ or $\mathrm{C}$ on pathologic examination. Compared with type $\mathrm{A}$, type $\mathrm{B}$ or $\mathrm{C}$ consist of many thick-walled vascular sinusoids, so it takes a longer time for them to achieve homogeneous enhancement. In addition, contrast-enhanced sequences by chance seized the transition from inhomogeneous to homogeneous enhancement. Therefore, they showed inhomogeneous enhancement on the first contrast-enhanced sequence and homogeneous enhancement on subsequent contrast-enhanced sequences. Now, it is not difficult to understand why the 6 type $\mathrm{B}$ or $\mathrm{C}$ lesions showed inhomogeneous enhancement on the first contrast-enhanced sequence and progressive contrast "filling in" on subsequent sequences. The reason why 2 lesions showed no progressive contrast "filling in" cannot be explained with their pathologic findings. However, it is noteworthy that they were the smallest among the 21 lesions. There is a possibility that it is difficult to determine their contrast "filling in" because of their small sizes. In addition, heterogeneous enhancement can manifest falsely as homogenous enhancement on some old-model MR scanners with out-of-date hardware and software. To determine the enhancement pattern, these factors should also be taken into consideration.

Two points about the difference between the intraparenchymal cavernous hemangiomas and the extra-axial ones are noteworthy. First, enhancement characteristics are different between the 2 lesions. The intraparenchymal cavernous hemangiomas usually demonstrate no or slight enhancement on conventional contrast-enhanced T1-weighted images, and delayed contrast-enhanced MR may exhibit diffuse contrast enhancement, ${ }^{14}$ whereas the extra-axial hemangiomas demonstrate markedly homogeneous enhancement or progressive contrast "filling in" on conventional contrast-enhanced images. Although both of them are vascular malformations composed of slowly perfused sinusoidal vessels, the difference in the rate of blood flow between the 2 lesions may be mainly responsible for their different enhancement characteristics. ${ }^{15,16}$ Second, T2*-gradient recalled-echo (GRE) sequences and susceptibility-weighted MR imaging, especially high-resolution susceptibility-weighted MR imaging, may show intralesional tubular structures that correspond to cavernous types of vessels in intraparenchymal cavernous hemangiomas. ${ }^{17}$ However, T2*-GRE sequences and susceptibilityweighted MR imaging of CSCHs have not been reported, and they may possibly provide us with additional useful information. ${ }^{18}$

Although progressive contrast "filling in" in cavernous sinus lesions on conventional contrast-enhanced sequences is not specific, it is suggestive of the diagnosis of CSCHs. ${ }^{19}$ As for lesions in which progressive contrast "filling in" cannot be ascertained, a postcontrast delayed MR image is suggested for further evaluation.

Differential diagnosis includes meningiomas, pituitary adenomas, neurilemomas, metastases, Tolosa-Hunt syndrome, intracavernous aneurysms, cavernous hemangiomas, lymphomas, teratomas, chondromas, chordomas, chondrosarcomas, lipomas, and dermoid cysts. According to their characteristic CT and MR findings, namely calcifications, fatty compositions, flow void, MR signal intensity features, and clinical data, it is not difficult to differentiate CSCHs from most of these lesions. ${ }^{20-24}$ However, angioblastic and syncytial meningiomas, neurilemomas, chondromas, chordomas, and chondrosarcomas may demonstrate hypointensity or isointensity on T1-weighted images and markedly homogeneous signal intensity on T2-weighted images because of lack of calcifications. $^{25-29}$ After contrast, they may display heterogeneous enhancement. On this condition, their radiologic findings are quite similar to those of type $\mathrm{B}$ or $\mathrm{C}$ of $\mathrm{CSCH}$ s. If contrast "filling in" in a lesion is observed, and on subsequent sequences the lesion enhances homogeneously, the diagnosis of CSCHs is highly suggested. ${ }^{30-33}$ Otherwise, the likelihood of CSCHs is less. However, if contrast "filling in" is observed, but the lesion does not enhance homogeneously on subsequent sequences, a postcontrast delayed MR imaging might be very helpful. Although chondromas and chordomas may enhance homogeneously on delayed MR imaging, they most likely take longer than $\mathrm{CSCH}$ to do so. ${ }^{13,34-36}$ According to this difference, CSCHs might be differentiated from chondromas and chordomas. Unfortunately, only 1 delayed MR imaging was performed in our series. On the other hand, they may demonstrate homogeneous enhancement. It is difficult to differentiate them from type A CSCHs. However, markedly homogeneous enhancement is more compatible with the diagnosis of $\mathrm{CHCH}^{10}$

Progressive contrast "filling in" was more frequently seen in our series, but in the literature, there is only 1 case report of $\mathrm{CSCH}$ about progressive contrast "filling in" on conventional contrast-enhanced MR imaging. ${ }^{29}$ In our opinion, 3 causes may account for this frequency in our study. In our routine MR examinations, fast contrast injection has been adopted, and the contrast material injection rate of $1.6 \mathrm{~mL} / \mathrm{s}$ is near bolus injection. However, no fast contrast material injection 
was reported in the literature. ${ }^{8,9,37,38}$ Second, in general, the MR scanners used in our series were superior to those used in the literature in both hardware and software. ${ }^{8,9,37,38}$ On the one hand, they provided us with high-quality MR images. On the other, they completed the process from advancing to scan to initiation of scan in less time after injection of contrast material. Although the perfusion of a cavernoma is slow, fast contrast material injection makes the amount of contrast agent entering a cavernoma increase within the first few minutes after administration, thereby making detection on conventional contrast-enhanced MR imaging easier. Also, fast MR scanning after injection of contrast material demonstrates earlier enhancement characteristics on conventional contrastenhanced MR imaging. Third, we paid more attention to this enhancing pattern.

According to our investigation, the current concept in the literature that type A lesions illustrate marked homogeneous enhancement, whereas type $\mathrm{B}$ or $\mathrm{C}$ lesions show heterogeneous enhancement is inaccurate. ${ }^{6,7}$ All of type A, B, and C lesions may show homogeneous enhancement after contrast. The real difference among them may be that type A lesions most likely show homogeneous enhancement earlier than the latter 2 on MR imaging. If so, a crude prediction of pathologic subtypes of CSCHs may be possible on the basis of this difference. However, there is only 1 type A case in our series, so statistical analysis cannot be performed. More cases with $\mathrm{CSCH}$ will be needed before a conclusion is drawn.

According to intraoperative findings, type A is spongelike with an intact pseudocapsule. It looks smooth and feels soft and elastic, with very high tension. Type B is mulberrylike with an incomplete or absent pseudocapsule. It looks granular and feels solid. Type C consists of both a mulberrylike and spongelike composition. Intraoperatively, type $\mathrm{B}$ or type $\mathrm{C}$ has a greater likelihood of being removed completely than type A. ${ }^{6-8}$ Therefore, contrast "filling in" on conventional contrast-enhanced MR imaging perhaps has significance for preoperative treatment planning and surgical prognosis of CSCHs. In the future, further study with dynamic MR or CT is needed to determine the time intervals for the 3 subtypes to achieve homogeneous enhancement, respectively.

In conclusion, progressive contrast "filling in" in the tumors on conventional contrast-enhanced MR images can aid in differentiating between cavernous sinus lesions and suggest the diagnosis of cavernous hemangiomas.

\section{Acknowledgments}

We thank Professor Luolin and his colleagues for great help in the processing of neuropathologic specimens.

\section{References}

1. Linskey ME, Sekhar LN. Cavernous sinus hemangiomas: a series, a review, and a hypothesis. Neurosurgery 1992;30:101-08

2. Biondi A, Clemenceau S, Dormont D, et al. Intracranial extra-axial cavernous (HEM) angiomas: tumors or vascular malformations? I Neuroradiol 2002;29:91-104

3. Peker S, Kilic T, Sengoz M, et al. Radiosurgical treatment of cavernous sinus cavernous haemangiomas. Acta Neurochir (Wien) 2004;146:337-41; discussion 340

4. Suri A, Ahmad FU, Mahapatra AK. Extradural transcavernous approach to cavernous sinus hemangiomas. Neurosurgery 2007;60:483-88; discussion 488-89

5. Ohata K, El-Naggar A, Takami T, et al. Efficacy of induced hypotension in the surgical treatment of large cavernous sinus cavernomas. J Neurosurg 1999;90:702-08
6. Zhou LF, Mao Y, Chen L. Diagnosis and surgical treatment of cavernous sinus hemangiomas: an experience of 20 cases. Surg Neurol 2003;60:31-36; discussion 36-37

7. Yao Z, Feng X, Chen X, et al. Magnetic resonance imaging characteristics with pathological correlation of cavernous malformation in cavernous sinus. J Comput Assist Tomogr 2006;30:975-79

8. Shi J, Hang C, Pan Y, et al. Cavernous hemangiomas in the cavernous sinus. Neurosurgery 1999;45:1308-13; discussion 1313-14

9. Lombardi D, Giovanelli M, de Tribolet N. Sellar and parasellar extra-axial cavernous hemangiomas. Acta Neurochir (Wien) 1994;130:47-54

10. Mendonca JL, Viana SL, Matsumine M, et al. Cavernous angioma of the cavernous sinus: imaging findings. Arq Neuropsiquiatr 2004;62:1004-07

11. Momoshima S, Shiga $\mathrm{H}$, Yuasa $Y$, et al. MR findings in extracerebral cavernous angiomas of the middle cranial fossa: report of two cases and review of the literature. AJNR Am J Neuroradiol 1991;12:756-60

12. Jeong MG, Yu JS, Kim KW. Hepatic cavernous hemangioma: temporal peritumoral enhancement during multiphase dynamic MR imaging. Radiology 2000;216:692-97

13. Shim YW, Chung TS, Kang WS, et al. Hemodynamical assessment of cavernous hemangioma in cavernous sinus using MR-DSA and conventional DSA. Yonsei Med J 2003;44:908-14

14. Thiex R, Kruger R, Friese S, et al. Giant cavernoma of the brain stem: value of delayed MR imaging after contrast injection. Eur Radiol 2003;13 Suppl 4:L219-25

15. Pinker K, Stavrou I, Knosp E, et al. Are cerebral cavernomas truly nonenhancing lesions and thereby distinguishable from arteriovenous malformations? MRI findings and histopathological correlation. Magn Reson Imaging 2006;24:631-37

16. Yamamoto $\mathrm{Y}$, Weining $\mathrm{Z}$, Ohashi $\mathrm{T}$. Intracavernous cavernous hemangioma: dynamic CT findings and effectiveness of irradiation-case report. Neurol Med Chir (Tokyo) 1992;32:93-95

17. Pinker K, Stavrou I, Szomolanyi P, et al. Improved preoperative evaluation of cerebral cavernomas by high-field, high-resolution susceptibility-weighted magnetic resonance imaging at 3 Tesla: comparison with standard $(1.5 \mathrm{~T})$ magnetic resonance imaging and correlation with histopathological findings-preliminary results. Invest Radiol 2007;42:346-51

18. Rauscher A, Sedlacik J, Barth M, et al. Magnetic susceptibility-weighted MR phase imaging of the human brain. AJNR Am J Neuroradiol 2005;26:736-42

19. Suzuki M, Matsui O, Ueda F, et al. Dynamic MR imaging for diagnosis of lesions adjacent to pituitary gland. Eur J Radiol 2005;53:159-67

20. Smith JK. Parasellar tumors: suprasellar and cavernous sinuses. Top Magn Reson Imaging 2005;16:307-15

21. Ruscalleda J. Imaging of parasellar lesions. Eur Radiol 2005;15:549-59

22. Pamir MN, Kilic T, Ozek MM, et al. Non-meningeal tumours of the cavernous sinus: a surgical analysis. J Clin Neurosci 2006;13:626-35

23. Cakirer S. MRI findings in Tolosa-Hunt syndrome before and after systemic corticosteroid therapy. Eur J Radiol 2003;45:83-90

24. Demir MK, Ozdemir H, Unlu E, et al. Differential diagnosis of spinal epidural meningioma and hemangioma at MR imaging. Radiology 2007;244:933-34

25. Pamir MN, Ozduman K. Analysis of radiological features relative to histopathology in 42 skull-base chordomas and chondrosarcomas. Eur J Radiol 2006;58:461-70

26. Leproux F, de Toffol B, Aesch B, et al. MRI of cranial chordomas: the value of gadolinium. Neuroradiology 1993;35:543-45

27. Allan CA, Kaltsas G, Evanson J, et al. Pituitary chondrosarcoma: an unusua cause of a sellar mass presenting as a pituitary adenoma. J Clin Endocrinol Metab 2001;86:386-91

28. de Tella OI Jr, Herculano MA, Prandini MN, et al. Chordoid meningioma: report of two cases. Arq Neuropsiquiatr 2003;61:91-94

29. Salanitri GC, Stuckey SL, Murphy M. Extracerebral cavernous hemangioma of the cavernous sinus: diagnosis with MR imaging and labeled red cell blood pool scintigraphy. AJNR Am J Neuroradiol 2004;25:280-84

30. Joo YG, Korogi $Y$, Hirai $T$, et al. Differential diagnosis of extra-axial intracranial tumours by dynamic spin-echo MRI. Neuroradiology 1995;37:522-25

31. Nagele T, Petersen D, Klose U, et al. Dynamic contrast enhancement of intracranial tumors with snapshot-FLASH MR imaging. AJNR Am J Neuroradiol 1993;14:89-98

32. Ikushima I, Korogi Y, Kuratsu J, et al. Dynamic MRI of meningiomas and schwannomas: is differential diagnosis possible? Neuroradiology 1997;39:633-38

33. Kothary N, Law M, Cha S, et al. Conventional and perfusion MR imaging of parafalcine chondrosarcoma. AJNR Am J Neuroradiol 2003;24:245-48

34. Ikushima I, Korogi Y, Hirai T, et al. Chordomas of the skull base: dynamic MRI. J Comput Assist Tomogr 1996;20:547-50

35. Tanohata K, Maehara T, Aida N, et al. Computed tomography of intracrania chondroma with emphasis on delayed contrast enhancement. J Comput Assist Tomogr 1987;11:820-23

36. Takenaka K, Nishimura Y, Andoh T, et al. Delayed postcontrast CT and MR imaging of chondroid chordoma-case report. Neurol Med Chir (Tokyo) 1992;32:28-31

37. Sohn $\mathrm{CH}, \mathrm{Kim} \mathrm{SP}, \mathrm{Kim}$ IM, et al. Characteristic MR imaging findings of cavernous hemangiomas in the cavernous sinus. AJNR Am J Neuroradiol 2003;24:1148-51

38. Katayama Y, Tsubokawa T, Miyazaki S, et al. Magnetic resonance imaging of cavernous sinus cavernous hemangiomas. Neuroradiology 1991;33:118-22 